\title{
A FIELD METHOD OF DETERMINING LONGITUDES BY OBSERVATIONS OF THE MOON.
}

A SHORT note appeared in these columns on A April 23 (p. 590) with reference to a valuable paper by Mr. E. B. H. Wade, published by the Survey Department of Egypt. The paper, however, is one showing so much originality, and the instrument and method appears to be of such value, that a more extended notice is called for, the more so as the paper in question is by no means generally accessible.

Mr. Wade considers the case of field survey operations where at least one point, and preferably more, should be determined independently by a direct astronomical method. Latitudes are easy enough, and longitudes would be if telegraphic connection with an observatory were available. Until wireless timesignals are distributed over the world which might be picked up with portable receiving apparatus, the observer has to rely upon his chronometers, which become cumulatively misleading, or upon lunar observations. These latter, Mr. Wade points out, may be divided into two classes-those which depend directly upon the rotation of the earth, of which moon culmination observations serve as an example, and those which do

PLATE "I.

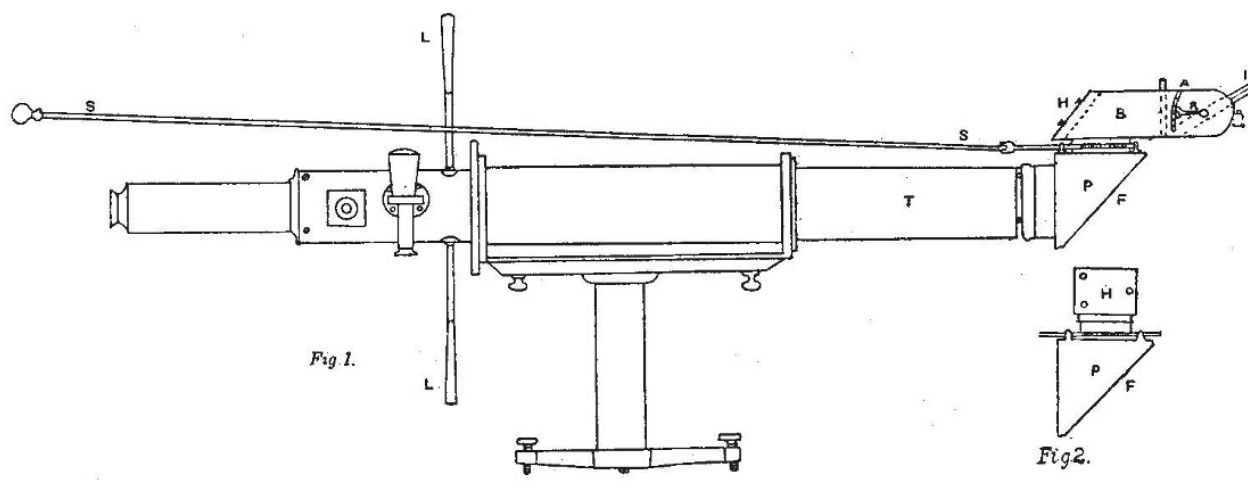

The telescope $T$ with its reflector $F$ is mounted as an altazimuth, the motion in altitude being given by the handles 1. $\mathrm{B}$, the box-sextant, containing the horizon mirror $\mathrm{H}$ and index mirror $\mathrm{I}$, is actuated by worm gear by means of the long ba the axis of the actual telescope. receives light from a third, which may be parallel with it or may be inclined at an angle of $10^{\circ}$ or so, roughly indicated by a pointer. The real point is that at whatever angle it is set, it may be clamped infallibly until the observations are complete. The box containing these two last-mentioned mirrors is capable of slow rotation by means of a worm from the eye end of the telescope, so that if, for instance, these two mirrors were set at an angle of $5^{\circ}$ and the worm were turned, the observer would see successively all points in a circle of $10^{\circ}$ radius round the point to which the unintercepted part of the instrument was directed. Supposing now the moon to be observed directly, and a following star, which is just too far away to be brought into contact with the limb, to be seen by double reflection, then a slight sweeping motion may be given so as to ascertain the exact time of tangential contact. Similarly a preceding star which is just too near for a tangential contact may, be observed until the moment at which this is seen also. Actually it is the star, not the moon, which is looked at directly, as by that means the moon's light after two reflections from unsilvered glass is sufficiently subdued to allow sixth-magnitude stars to be observed in contact. The designer has ingeniously introduced the equivalent of the spider lines of a transit instrument by providing a slightly prismatic plate which may be set in any one of three positions in one of the optical paths, thus making small but invariable differences in the apparent angle, so that three observations are possible for each star.

It will be seen, then, that the accuracy obtainable is only limited by the optical definition of the telescope.

$\mathrm{Space}$ is not available for follow- not, for instance, lunar distances and occultations, and these have the advantage of being about thirty times less sensitive to errors of time.

Mr. Wade has set himself to devise a method of making lunar distance observations with an accuracy outside the possible range of the sextant with apparatus that is easily portable. He refers to 'Talcott's method of determining latitude by observations of the equidistance of the zenith from two stars as compared with the actual measurement of its distance from a particular star. "In quite the same way" he suppresses " the graduated circle of the ordinary sextant and finds the moon's apparent position from the condition that it is equidistant from two or more stars, one of which, of course, must precede and the other follow the moon." By suppressing the graduated circle he removes the principal obstacle to the evolution of the sextant.

In order to arrive at this result, he starts with a good telescope of $2 \frac{1}{2}$ inches aperture and magnifying 40 diameters, carrying a reflector set at $45^{\circ}$ in front of the object-glass. The telescope is always horizontal, and can turn upon its longitudinal axis as well as upon its stand in azimuth. It therefore commands all points of the sky, and the observer can sit in comfort, a point essential for accuracy. The view of half the objectglass is intercepted by a second mirror set at $45^{\circ}$, which ing the author of the paper through his discussion of the observations or for referring to his excellent graphic methods for shortening the calculations where they are available. It is sufficient to say that with his apparatus he has found that the probable error of a single observation is of the order of $\mathrm{I}^{\prime} 5$ seconds of longitude.

C. V. Boys.

\section{THE CAVENDISH LABORATORY.}

N October Io, I8jo, the seventh Duke of Devonshire wrote to the then Vice-Chancellor of the University of Cambridge, offering to provide funds for a building and for apparatus for the teaching of experimental physics. The building was opened on June 16,1874 , when the Chancellor received the thanks of the University, and at the same time expressed his "wish to provide all instruments for the Cavendish Laboratory which Prof. Maxwell may consider to be immediately required either in his lectures or otherwise."

Twenty years later the laboratory was increased towards the south by a new building, running along Free School Lane, which contained, amongst other rooms, a spacious elementary laboratory. For this

$$
\text { NO. 20I6, VOL. 78] }
$$

